\title{
Artefakte im Altquartär von Obergaliläa (Nordisrael)
}

\author{
KARl BRUNNACKeR, WOlFGANG BOENIGK, GERSHON BRUder, \\ Gabriele G. Hahn, Avraham Ronen \& Wolfgang Tillmanns*)
}

Stratigraphy, carbonate rocks, basalts, soil profiles, size distribution, heavy minerals, paleomagnetism, inverse magnetization, lower Pleistocene, Acheulian, artefacts, Israel, Galilee

\begin{abstract}
Kurzfassung: Bei Yiron treten auf Karbonatgesteinen der Oberkreide und des Alttertiärs tonige Bodensedimente auf. Lokal ist darin ein Kalk- und Flintschotter eingeschaltet, der altpaläolithische Artefakte führt. Ein inzwischen erosiv aufgelöster revers magnetisierter Deckbasalt bildet das Hangende. Er ist zum damals bereits angelegten Jordan-Graben hin orientiert. Die Artefakte müssen demnach älter sein als die Brunhes-Matuyama-Grenze. Sie gehören neben den Funden von Evron (nördlich Haifa) und Ubaidiya (JordanGraben) zu den ältesten Hinterlassenschaften des Menschen im Vorderen Orient.
\end{abstract}

Ferner liegt eine ebenfalls relativ alte Faustkeil-Kultur auf den Plateaus von Yiron und Baram. Die Plateaus von Yiron und Baram lassen sich in geomorphologischer Hinsicht als ein flaches Hochtal interpretieren. Die weitere Entwicklung ist durch eine Kerbtal-Bildung gekennzeichnet.

\section{[Old Quaternary Artefacts from Higher Galilea (Northern Israel)]}

Abstract: Near Yiron clayey soil sediments occur on upper cretaceous and early tertiary carbonate rocks. Within these soil sediments there are locally intercalations of limestone and flint gravels containing old palaeolithic artifacts. The overlying layer is an erosively dissolved basalt cover displaying reverse magnetism. It is oriented to the Jordan Graben which already existed at that time. From this can be inferred that the artefacts which occur in the layers underlying the basalt have to be older than the BrunhesMatuyama boundary. These artefacts as well as those from Evron (north of Haifa) and Ubaidiya (Jordan Graben) are among the oldest remains of human activity in the Near East.

*) Anschrift der Autoren: Prof. Dr. W. Boenigk, Prof. Dr. K. BrunNaCker, Dr. G. G. Hahn, Priv.-Doz. Dr. W. TillmanNs, Geologisches Institut der Universität zu Köln, Zülpicher Straße 49, D-5000 Köln 1. G. BRUDER, Kibbuz Baram, Israel. Prof. Dr. A. Ronen, Universität Haifa, Mount Carmel, Haifa 31 999, Israel.
Another handaxe assemblage has been found on the Plateaus of Yiron and Baram. Geomorphologically, these plateaus can be interpreted as a flat high valley. The further development is characterized by a V-shaped valley formation.

\section{Vorbemerkungen}

In Obergaliläa liegen rd. $15 \mathrm{~km}$ westlich des Jordan-Grabens nächst der Grenze zum Libanon Basalt-Plateaus im Umfeld von Yiron. Auf diesen Plateaus wurden Artefakte einer alten Faustkeil-Kultur gefunden (RONEN et al. 1974). Ursprüngliches Ziel war, durch geologische und geomorphologische Untersuchungen Vorstellungen vom Alter der Kultur zu entwickeln. Nachdem Artefakte jedoch auch in einem Schotter im Liegenden des Basalts gefunden wurden (RONEN et al. 1980), hatte sich das Schwergewicht der Fragen auf diese älteren Kulturreste verschoben (Abb. 1).

Ermöglicht wurden die Arbeiten durch die finanzielle Unterstützung der Volkswagen-Stiftung, der wir an dieser Stelle danken möchten.

Die paläomagnetischen Untersuchungen wurden mit Mitteln des Bundesministers für Forschung und Technologie unter dem Förderkennzeichen KF 2003/3 unterstützt.

\section{1. Überblick}

Der Untergrund des bearbeiteten Gebietes besteht aus Kalken der Oberkreide und des ältesten Tertiärs, bei denen nach dem Hangenden hin der mergelige Charakter zunimmt. In diese Kalke sind Flintknauern und - schmitzen eingeschaltet, die fluviatil aufgearbeitet das Rohmaterial für die Werkzeuge geliefert haben (Abb. 1 u. 2). Tektonisch handelt es sich um eine Muldenposition mit Streichen von SE nach NW. Die Achse der Mulde verläuft über Yiron (Abb. 2). 


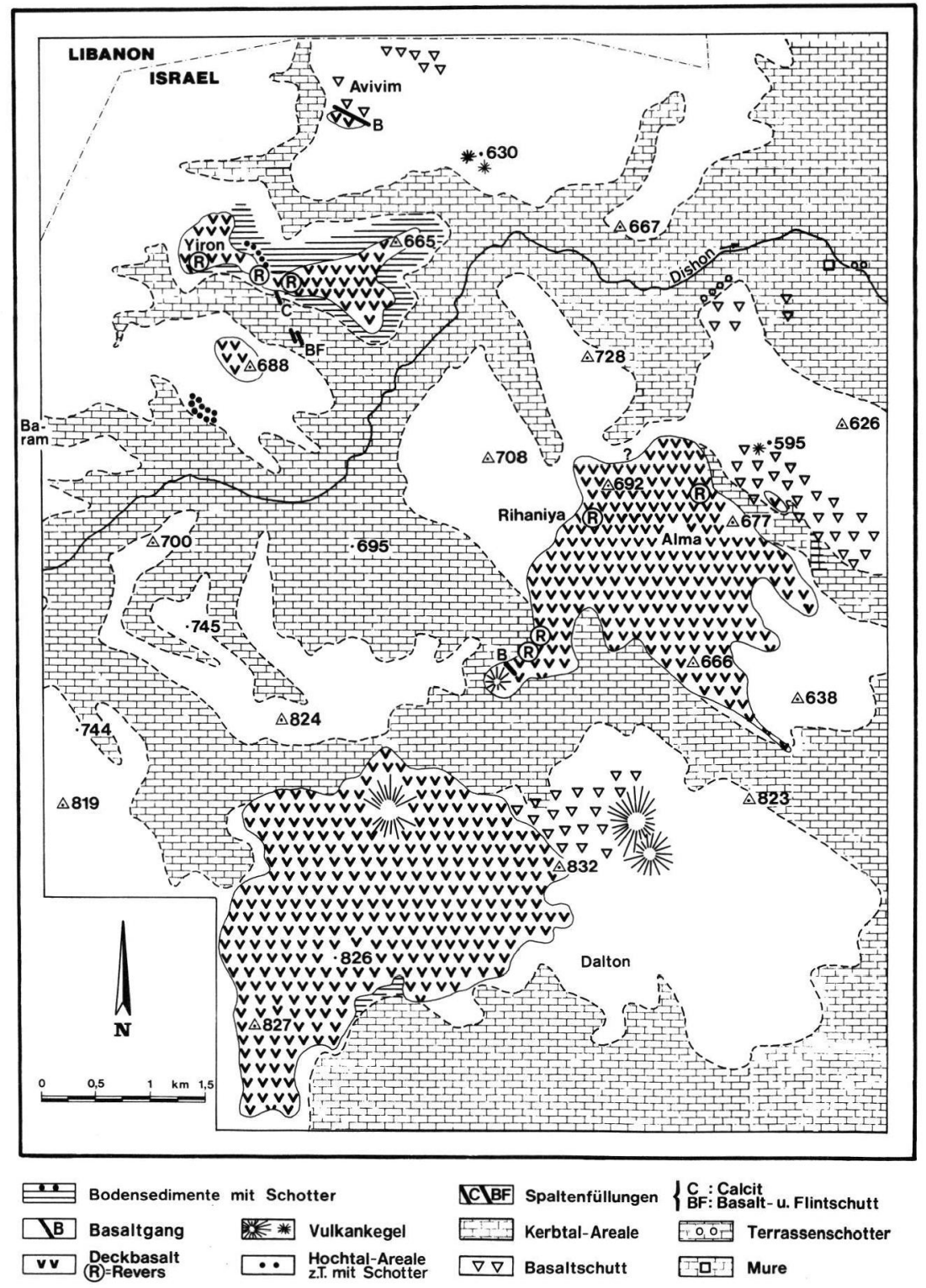

Abb. 1: Geologisch-geomorphologische Übersicht von Ober-Galiläa.

Fig. 1: Geological-geomorphological survey of Upper Galilee.

Darüber folgen besonders östlich von Yiron Terraecalcis-Sedimente (Abb. 2), in die stellenweise Schotter eingeschaltet sind. Überdeckt wird die Abfolge durch einen infolge Erosion in Reste aufgelösten PlateauBasalt auf dem bzw. in dessen Nachbarschaft nordöstlich von Baram und östlich von Yiron zahlreiche Artefakte liefen (Acheuléen).

Anschließend kam es zur Eintiefung des Dishon- und Avod-Tales, die zum Jordan-Graben entwässern.
Die Oberfläche der bis $10 \mathrm{~m}$ dicken Basaltdecke liegt bei Yiron (und Baram) etwas unter $700 \mathrm{~m} \mathrm{NN}$. Die südlichen Deckenreste, soweit sie außerhalb der Muldenposition erhalten sind, liegen um $820 \mathrm{~m} \mathrm{NN}$ (Abb. 1).

An den Hängen wurde eiszeitlicher (?) Frostschutt kaum beobachtet. Bereichsweise hat die Basaltdecke jedoch erhebliche Mengen an Blockwerk geliefert, die die Gehänge überkleiden. 


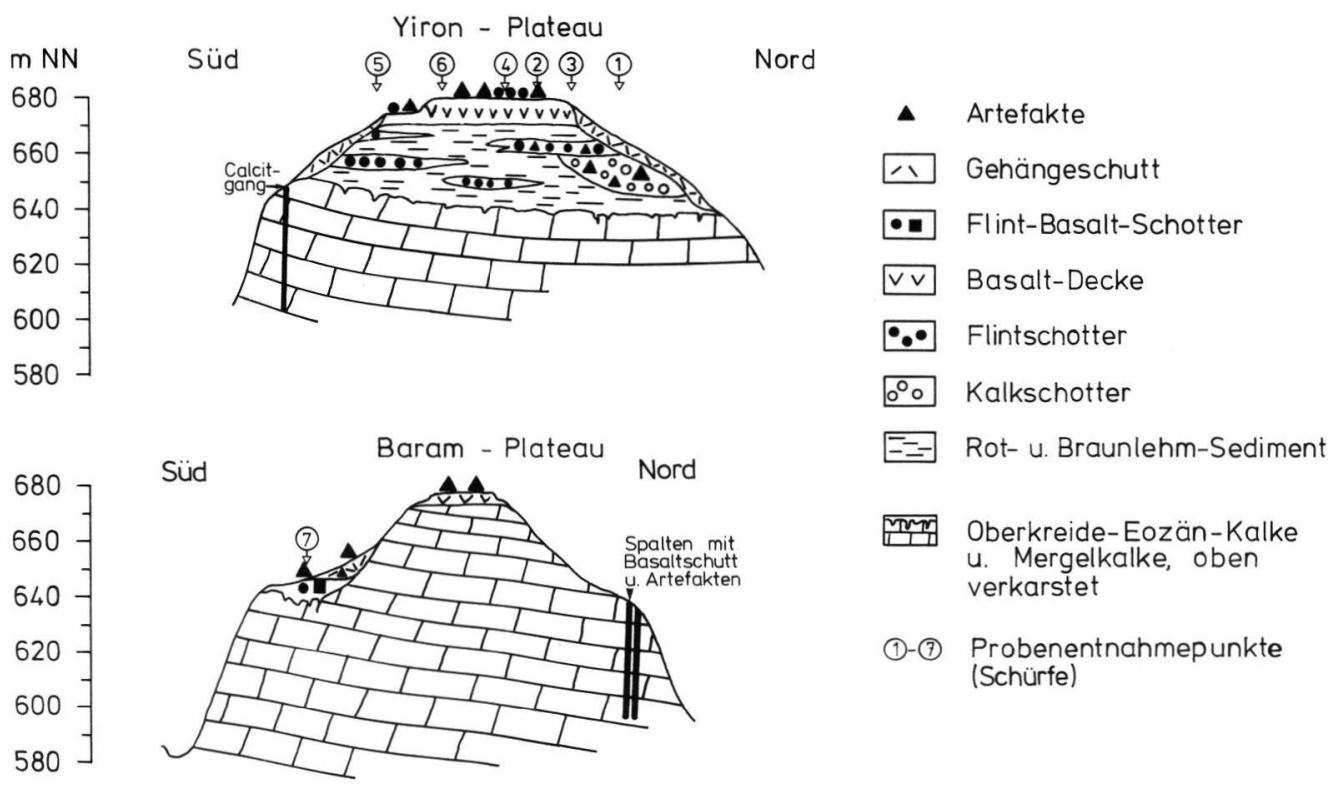

Abb. 2: Profilaufbau bei Yiron und Baram.

Fig. 2: Profile section near Yiron and Baram.

Klimatisch handelt es sich heute im Vergleich zur Küstenzone und zum Jordangraben um eine relativ feucht-kühle Gegend mit einem Jahresniederschlag um $800 \mathrm{~mm}$ und einer jährlichen Durchschnittstemperatur von $16,4^{\circ} \mathrm{C}$ (Ramala: $760 \mathrm{~m} \mathrm{NN}$ ).

\section{Geologie}

Das Liegende bilden mergelige Kalkgesteine mit Flinteinschaltungen. Darüber folgen z. T. Bodensedimente, die von einer über die Mulde von Yiron hinausreichenden Basaltdecke überlagert werden.

\subsection{Bodensedimente}

In die östlich von Yiron liegenden, bis $35 \mathrm{~m}$ mächtigen Bodensedimente sind Kalk-Flint- und FlintSchotter als lokale Bildungen eingelagert. Die Artefaktführung des Schotters unmittelbar östlich von Yiron gab den Anstoß, diese Terrae-calcis-Sedimente vor allem im hangenden Bereich eingehender $\mathrm{zu}$ untersuchen. Dazu wurde eine Reihe von Baggerschürfen angelegt (Abb. 2 u. 3).

Profil 1 (Yiron, Kiesgrube nächst Schotterbasis):

$120 \mathrm{~cm}$ Kalkschotter, mit rötlich-braunem, tonigen Sand als Zwischenmittel, Konglomerat

$190 \mathrm{~cm}$ Rotbrauner, sandiger Ton; oben zentimeterdicke tonige Sand-Schmitzen und einzelne KarbonatGerölle (bis $1 \mathrm{~cm} \varnothing$ ); im dm-Bereich grauschlierig; unten Eisen-Mangan-Konkretionen (1 mm $\varnothing)$, auf Klüften schwache Mangan-Ausscheidungen
Profil 2 (Yiron; zwischen Flint-Schotter und BasaltUntergrenze, $50 \mathrm{~m}$ von der Straße und $140 \mathrm{~m}$ vom Basaltrand entfernt):

$150 \mathrm{~cm}$ Basaltschutt

$60 \mathrm{~cm}$ Rotlehm, Ton, schluffig, geröllfrei

$20 \mathrm{~cm}$ Übergang von Braunlehm zu Rotlehm, braun- und rotfleckig

$120 \mathrm{~cm}$ Braunlehm, Ton, schwach schluffig, gelblich, unten mit grauen Flasern ( $10 \mathrm{~cm}$ dick), einzelne Flintund verwitterte Karbonatgerölle

$30 \mathrm{~cm}$ Flintschotter

Profil 3 (Yiron)

$160 \mathrm{~cm}$ Braunlehm, Ton, schwach sandig, gelblich-braun gefleckt, mit einzelnen Basaltbrocken, etwas Flintgerölle

$15 \mathrm{~cm}$ Flintschotter, Feinkies (5 mm $\varnothing$ ), sandig-lehmig, grau, stark eisen-mangan-fleckig

$180 \mathrm{~cm}$ Flintschotter, fein bis grob, rotbraunes, toniges Zwischenmittel, Gerölle mit $\varnothing$ bis $15 \mathrm{~cm}$

Profil 4 (Yiron; Südrand des Plateaus, rd. $10 \mathrm{~m}$ unterhalb der Basaltuntergrenze):

$100 \mathrm{~cm}$ Schuttdecke, mit sehr viel Flintmaterial und wenig Basaltschutt

$150 \mathrm{~cm}$ Ton, schwach schluffig, rotbraun, mit einzelnen Flintgeröllen, mangan-fleckig

$100 \mathrm{~cm}$ Flintschotter, mit rotbraunem, lehmigen Zwischenmittel 


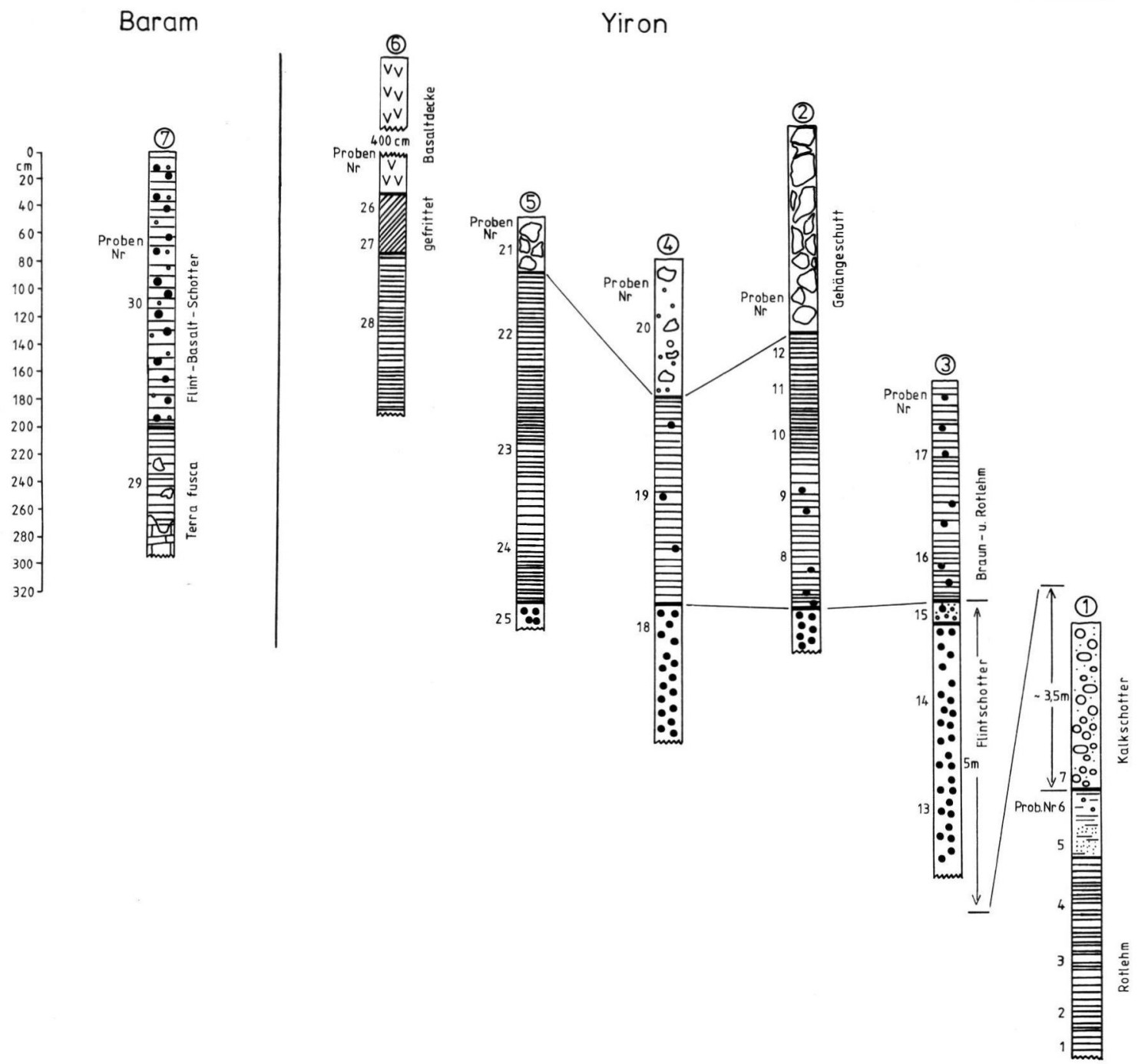

Abb. 3: Profile in den präbasaltischen Bodensedimenten bei Yiron und dem Hochtalboden bei Baram (vgl. Abb. 2).

Fig. 3: Profiles in the prebasaltic soil sediments near Yiron and the high-valley soil near Baram (cf. fig. 2).

Profil 5 Südseite des Plateaus (Yiron; nächst Basaltkontakt):

$40 \mathrm{~cm}$ Gehängeschutt

$80 \mathrm{~cm}$ Rotlehm, Ton schluffig, schwach sandig, mit etwas Basaltschutt

$90 \mathrm{~cm}$ Rotlehm, Ton, stark schluffig, mit bräunlichen Tapeten, Mangan-Flecken, etwas graufleckig

$70 \mathrm{~cm}$ Rotlehm, Schluff, stark tonig, mit roten Tontapeten

$20 \mathrm{~cm}$ Flintschotter mit schluffigem Zwischenmittel

Profil 6 (Yiron; Basaltkontakt südlich Yiron): $500 \mathrm{~cm}$ Basalt

$40 \mathrm{~cm}$ gefritteter Rotlehm, Grobsand, schluffig, tonig $120 \mathrm{~cm}$ Rotlehm, Schluff, sandig, tonig
Profil 7 (südlich Baram-Plateau):

$200 \mathrm{~cm}$ Flintlehm, dunkelgrau-braun, Flintgerölle bis $(30 \mathrm{~cm} \varnothing)$, einzelne Basaltgerölle (bis $5 \mathrm{~cm} \varnothing$, das Zwischenmittel ist ein stark sandiger Ton

$70 \mathrm{~cm}$ Braunlehm, Ton, hellbraun mit angelöstem Kalkstein

Liegendes Kalkstein, verkarstet

Die Bodensedimente sind auf den Bereich östlich Yiron konzentriert (kombiniertes Profil):

bis $10,0 \mathrm{~m}$ Basaltdecke

z.T. 0,4 m gefritteter Rotlehm

5,0 m Rotlehm (z. T. Braunlehm), mit einzelnen Artefakten

5,0 m Flintschotter, mit Artefakten, schlecht sortiert 
15,0 m Kalkschotter, mit Artefakten, schlecht sortiert, tonig-sandiges $\mathrm{Z}$ wischenmittel

bis $5,0 \mathrm{~m}$ Rotlehm (z. T. Braunlehm)

Liegendes Kalkstein, verkarstet
Lokale Flintschotter kommen innerhalb der Bodensedimente auch sonst vor. Diese Sedimentfolge erweist sich damit als eine Bildung, welche die Muldenzone bei Yiron nachzeichnet, wenngleich auch andernorts bisweilen Bodensedimente mit bis zu einigen Metern Mächtigkeit auftreten können.
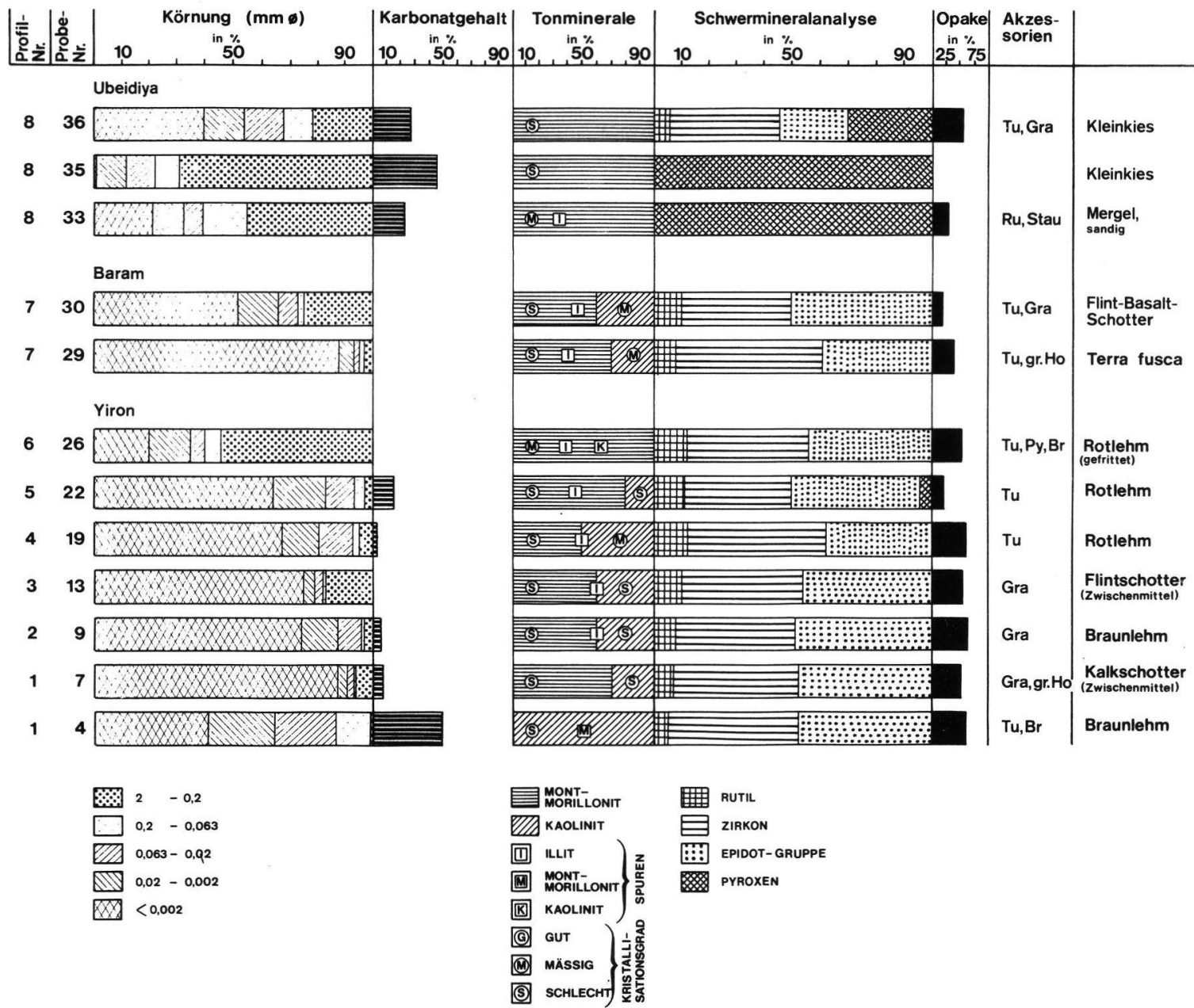

Abb. 4: Analytische Daten zu den Profilen aus Abb. 3 (Auswahl) und von Ubaidiya (Auswahl).

Fig. 4: Analytical data concerning the profiles in fig. 3 (selection) and of Ubaidiya (selection).

Laboruntersuchungen, die von Dipl.-Geol. ERVIN ISKANDER (1985) durchgeführt worden sind, haben folgendes erbracht (Abb. 4).

In nahezu allen analysierten Proben heben sich pedogene Lehme aufgrund der spezifischen Kornverteilung und des relativ hohen Karbonat-Gehaltes sowohl von den fluviatilen Schottern als auch von den Hangschutten ab. Die Kornverteilung der Lehme ist durch eine Dominanz der Schluff- und Tonfraktion charakterisiert.
Der hohe Tonanteil der Rotlehme steht nach ZOHARY (1959) mit der petrographischen Beschaffenheit der Muttergesteine in Zusammenhang. In Anlehnung an FLEXNER (1964) darf angenommen werden, daß die Muttergesteine der Rotlehme, die in der Umgebung anstehen, senone Kalke sind.

Die qualitative Tonmineralführung der untersuchten Proben zeigt im allgemeinen eine Montmorillonit/Kaolinit-Assoziation, wobei der Montmorillonit-Gehalt in nahezu allen Proben quantitativ überwiegt. 
Eine Änderung der Tonmineralführung in der stratigraphischen Abfolge läßt sich nicht feststellen.

Bemerkenswert ist der Zusammenhang zwischen dem Anstieg des Montmorillonit-Gehaltes und dem Auftreten von Pyroxenen in den postbasaltischen Proben. Es wäre denkbar, daß der Montmorillonit-Gehalt der präbasaltischen Lehmproben auf verwitterte Basalte zurückzuführen ist. Die in Frage kommenden Muttergesteine könnten miozäne Basalte sein, die im Raum Qarne-Hittim am Galiläa-See von pliozänen Sedimenten überlagert werden (vgl. OPPENHEIM 1962). In der Schwermineralfraktion $(0,063-0,4 \mathrm{~mm} \varnothing)$ dominieren bei den transparenten Mineralen Zirkon und die Epidot-Gruppe. Untergeordnet tritt Rutil auf. Turmalin, Granat, Brookit, grüne Hornblende und Titanit kommen in den Proben nur akzessorisch vor. Der Anteil der opaken Körner liegt generell über $50 \%$ am Gesamtspektrum der Schwerminerale.

Die in den Rotlehmen auftretenden Schwerminerale zeigen an, daß sie z. T. aus weiter entfernten Liefergebieten stammen. Eine Vermischung von fluviatil herantransportiertem Material mit einer äolischen Komponente läßt sich aufgrund der Korngrößenverteilung vermuten. Eine äolische Zufuhr wird auch von ZOHARY (1959) und HOROWITZ (1979) in Erwägung gezogen.

Die möglichen, primären Liefergebiete der in den Rotlehmen auftretenden Schwerminerale sind einmal die in Transjordanien anstehenden nubischen Sandsteine und zum anderen das Einzugsgebiet des Ur-Nils.

Die oberkretazischen nubischen Sandsteine zeigen nach VROMAN (1944) und GREENBERG (1960) ausschließlich ein stabiles Schwermineralspektrum (Zirkon, Rutil, Turmalin). Die Schwerminerale wurden über fluviatile Wege ins Cisjordanland verfrachtet. Ein westwärts gerichtetes, paläogenes Entwässerungssystem ist bis in den Grenzbereich Oligozän/Miozän zu verfolgen (NEEv, 1960). Mit der beginnenden Senkung des Jordan-Grabens zu Beginn des Miozäns wurde das genannte Entwässerungssystem unterbrochen.

Andererseits lieferte der (Ur-) Nil seit dem Oligozän neben Hornblende vorwiegend instabile (EpidotGruppe) und metamorphe (Staurolith, Disthen) Schwerminerale. Der Lauf des alten Nils kann entlang der Küstenlinie bis auf die Höhe von Tel Aviv nachgewiesen werden. Aufgrund der Schwermineralführung der Bohrung "Jaffa-1" südlich von Tel Aviv mit der Epidot-Gruppe, Pyroxen, Amphibol (Hornblende), Disthen, Staurolith, Zirkon, Rutil und Turmalin ist ein Zusammenfluß des alten Nils mit Flüssen, die einem E-W gerichteten Entwässerungssystem angehörten, im Grenzbereich Oligozän/Miozän nicht auszuschließen (vgl. NACHMIAS 1969). Aus den entlang der Küste nach $\mathrm{N}$ verfrachteten NilSedimenten wurden Schluff und die entsprechenden Schwerminerale ausgeweht und nach E transportiert (BOENIGK et al. 1985) und treten als äolische Komponente in den Ablagerungen in Obergaliläa auf.

Das Zwischenmittel der präbasaltischen Schotter zeigt das gleiche Schwermineralspektrum wie die Bodensedimente.

\subsection{Deckbasalt}

Im Arbeitsgebiet sind Reste von Basaltdecken mit maximal $10 \mathrm{~m}$ Mächtigkeit überliefert. Die Oberfläche der Platte westlich Dalton liegt um 820 m NN. Die Vorkommen von Alma und Yiron liegen zwischen $640 \mathrm{~m}$ und $690 \mathrm{~m}$ N. Diese Vorkommen zeigen also eine allgemeine Absenkungstendenz zur Muldenachse mit dem höchsten Absenkungsbetrag im E. Ein Basaltstrom östlich Alma floß zum Jordan-Graben. Er sinkt bis auf $615 \mathrm{~m} \mathrm{NN}$ ab, ein Zeichen dafür, daß dieser Graben bereits angelegt war.

Die Basalt-Decke ist in sich weitgehend ungegliedert. Lediglich bei Alma ist eine dm-dicke Lehm-Lage mit einigen Flint-Stücken zwischengeschaltet. Auch im Kibbuz Yiron deutet sich eine Zweiteilung an.

Vermutlich bildete der Basalt ursprünglich eine zusammenhängende Platte, die im Muldenbereich nachträglich etwas verstellt und durch Erosion aufgelöst wurde.

Gangfüllungen bei Avivim und südwestlich von Alma sowie aufgesetzte, bis $50 \mathrm{~m}$ hohe Schlackenkegel geben Hinweise darauf, daß es sich um eine von den Golan-Höhen unabhängige Förderung handelte. Andeutungsweise häufen sie sich an den Flanken der Mulde von Yiron.

Eine zweite, sehr schwache Förderungsphase wird durch zwei benachbarte Vulkanreste im Hochtal östlich von Yiron in $630 \mathrm{~m}$ NN angezeigt.

\subsection{Paläomagnetisches Verhalten des Deckbasaltes}

Aus dem Profil Rihaniya konnten aus 4 Einheiten 20 Einzelkerne, aus dem Profil Yiron 3 Einheiten mit 30 Einzelkernen und aus dem Profil Kibbuz Yiron 3 Einheiten mit 18 Einzelkernen entnommen werden. Im Labor des Geologischen Institutes Köln erfolgten mit Spinnermagnetometer MS-1 Minispin der Fa. MOLSPIN (Newcastle, England) die Messungen der natürlichen remanenten Magnetisierung (NRM) an insgesamt 68 Einzelkernen. 
Abb. 5 zeigt die ermittelten Magnetisierungsrichtungen $(\mathrm{DR} / \mathrm{IR}=$ Deklination rotiert/Inklination rotiert) aller gemessenen Proben als Durchstichpunkte ihrer Vektorenendpunkte im SCHMIDTschen Netz. In der Tabelle 1 sind die berechneten mittleren Magnetisierungsrichtungen der einzelnen Handstücke aus den Einzelkernen (SVKE), sowie die zugehörigen statistischen Parameter und die mittleren Intensitäten der Proben aufgelistet.

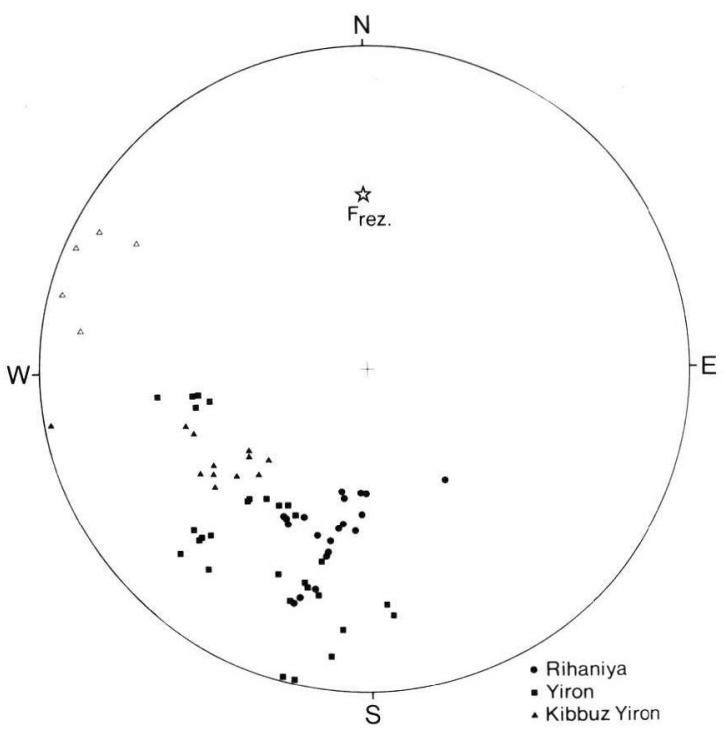

Abb. 5: Die Richtungen der natürlichen remanenten Magnetisierung aller Proben als

Durchstichspunkte ihrer Vektorenendpunkte.

Fig. 5: The directions of the natural remanent magnetisation of all samples

as perforation points of their vector ending points.

Geschlossene Symbole: negative Richtungen in der unteren Halbkugel

Closed symbols: negative directions in the inferior sphare

Offene Symbole: positive Richtungen in der unteren Halbkugel

Open symbols: positive directions in the inferior sphare.

Mit nach $\mathrm{S}$ weisenden Deklinationen und Inklinationswinkeln um $-40^{\circ}$ bilden die Remanenzvektoren nahezu aller Proben aus den Profilen Rihaniya und Yiron eine Magnetisierungsrichtung ab, die der Richtung des heutigen normalen Erdmagnetfeldes in Israel (359/45.5) \pm entgegengerichtet ist. Sie repräsentiert somit die Richtung eines revers polarisierten Erdmagnetfeldes.

Aufgrund des Verwitterungszustandes einiger Proben aus den Profilen Yiron und Kibbuz Yiron war eine sekundäre Überprägung durch das rezente Erdmagnetfeld zu erwarten. Mit einer mittleren Magnetisierungsrichtung von DR/IR 286/09 der Proben aus dem oberen Profilbereich Kibbuz Yiron und mit DR/IR 260/-45 aus Proben eines Handstückes aus Yiron zeichnet sich diese Überprägung deutlich ab. Die Remanenzvektoren der Proben aus den unteren Profilmetern Kibbuz Yiron weichen mit DR/IR 232/-45 nur wenig von der reversen Paläofeldrichtung ab (Abb. 5).

Es bleibt zu diskutieren, ob die reverse Polarität des Basaltes während der Matuyama-Epoche entstand oder lediglich einen Event oder eine Exkursion in der Brunhes-Epoche widerspiegelt. Für die erste Deutung sprechen das Alter von $600.000-650.000$ Jahren, das für den benachbarten Yarmouk-Deckenbasalt bestimmt wurde und die Einstufung der Artefakte im Liegenden des Basaltes als Pre-Late-Archeuleen (RONEN et al. 1980). Somit müssen die Basalte älter als die Brunhes-Matuyama-Grenze und damit älter als 690.000 Jahre sein.

\subsection{Deckbildungen}

Auf dem Basalt-Plateau von Yiron und darüberhinaus greifend liegen bei Baram die Faustkeil-Kulturen, welche ursprünglich Anlaß der Untersuchungen waren. Aus dem Basalt hat sich inzwischen eine $50 \mathrm{~cm}$ tiefe Braunerde (Erubasboden im Sinne von STREMME sen.) gebildet. Bei Yiron kommen stellenweise auch Überstreuungen von bis $1 \mathrm{~cm}$ großen Flintgeröllchen vor, wie sie auch im Profil 3, Probe 15, also in einer älteren Position gefunden wurden.

Eine Besonderheit bilden östlich von Yiron Knauern mit 5 bis $12 \mathrm{~cm} \varnothing$, deren Oberfläche eine starke Strukturierung („Blumenkohl”) aufweist. Im Inneren sind mm-große Quarzkristalle entwickelt, die zu einem zentralen Hohlraum hin orientiert sind. Herr SECK vom Mineralogisch-Petrographischen Institut unserer Universität hat eine dieser Knollen untersucht: „Sie ist aus einzelnen großen Quarzkörnern aufgebaut, die mit kleinen Einschlüssen regelrecht durchsetzt sind. Teilweise haben die Quarzkörner einschlußfreie Säume. Teils ist der Quarz auch rechtwinklig in Hohlräumen gewachsen. Bei den feinkörnigen Einschlüssen handelt es sich nach einer röntgenographischen Übersichtsaufnahme um Anhydrit, der durch seine drei stärksten Linien im Röntgendiagramm vertreten ist."

Genese und Herkunft dieser merkwürdigen Knollen müssen vorerst offen bleiben. Zu diskutieren wäre, ob es sich um Wurf- oder Schleudersteine des paläolithischen Menschen handelt.

Basalt-Restschutt wurde in Abb. 1 nur dort eingetragen, wo er in größeren Anreicherungen auftritt. 
Tab. 1: Die berechneten mittleren Magnetisierungsrichtungen aller Kerne einer Einheit (SVKE) und die dazugehörigen statistischen Parameter $\alpha_{95}$ und $\mathrm{K}$.

$\alpha_{95}$ ist der Fehlerkreis mit dem Radius $\alpha_{95}$ um den Mittelwert, innerhalb dessen sich mit $95 \%$ iger Wahrscheinlichkeit der wahre Mittelwert befindet.

$\mathrm{K}$ ist ein Präzisionsparameter, der ein Maß für die Streuung angibt.

Tab. 1: Directions of magnetisation.

\begin{tabular}{|c|c|c|c|c|c|c|}
\hline Profil & $\begin{array}{l}\text { Anzahl der beprobten } \\
\text { Einheiten }\end{array}$ & $\begin{array}{c}\text { Anzahl der gemessenen } \\
\text { Kerne }\end{array}$ & $\alpha_{95}$ & K & DR/IR (SVKE) & $\begin{array}{l}\text { mittl. Intensitäten } \\
\mathrm{A} / \mathrm{m}\end{array}$ \\
\hline Rihaniya & $\begin{array}{l}1 \\
2 \\
3 \\
4\end{array}$ & $\begin{array}{l}4 \\
6 \\
6 \\
4\end{array}$ & $\begin{array}{r}5.6 \\
6.2 \\
10.7 \\
7.8\end{array}$ & $\begin{array}{r}269 \\
118 \\
40 \\
139\end{array}$ & $\begin{array}{l}184 /-55 \\
203 /-51 \\
187 /-49 \\
196 /-32\end{array}$ & $\begin{array}{l}499 \times 10^{-2} \\
672 \times 10^{-2} \\
308 \times 10^{-2} \\
329 \times 10^{-2}\end{array}$ \\
\hline Yiron & $\begin{array}{l}1 \\
2 \\
3 \\
4 \\
5\end{array}$ & $\begin{array}{l}6 \\
6 \\
6 \\
6 \\
6\end{array}$ & $\begin{array}{r}4.3 \\
3.9 \\
4.1 \\
13.0 \\
4.7\end{array}$ & $\begin{array}{r}245 \\
290 \\
267 \\
27 \\
206\end{array}$ & $\begin{array}{l}224 /-27 \\
216 /-48 \\
262 /-45 \\
186 /-14 \\
197 /-32\end{array}$ & $\begin{array}{l}30 \times 10^{-1} \\
47 \times 10^{-1} \\
50 \times 10^{-1} \\
73 \times 10^{-1} \\
78 \times 10^{-1}\end{array}$ \\
\hline $\begin{array}{l}\text { Kibbuz } \\
\text { Yiron }\end{array}$ & $\begin{array}{l}1 \\
2 \\
3\end{array}$ & $\begin{array}{l}6 \\
5 \\
6\end{array}$ & $\begin{array}{r}6.5 \\
6.8 \\
13.7\end{array}$ & $\begin{array}{r}105 \\
129 \\
24\end{array}$ & $\begin{array}{l}237 /-51 \\
239 /-43 \\
286 / 09\end{array}$ & $\begin{array}{r}69 \times 10^{-1} \\
73 \times 10^{-1} \\
256 \times 10^{-1}\end{array}$ \\
\hline
\end{tabular}

Tab. 2: Schwermineral-Gehalt von Schottern und einer Mure am Hang des Dishon-Kerbtales.

Tab. 2: Heavy mineral content in the Dishon valley.

\begin{tabular}{|c|c|c|c|c|c|c|c|c|c|c|}
\hline Probe & & Epidot-Gr. & Zirkon & Rutil & Turmalin & Pyroxen & Granat & Staurolith & Anatas & Opake \\
\hline Mure & $\begin{array}{l}\text { Lehm } \\
\text { (gelb) }\end{array}$ & 28 & 44 & 10 & - & 8 & 1 & - & - & 74 \\
\hline Mure & $\begin{array}{l}\text { Lehm } \\
\text { (rot) }\end{array}$ & 18 & 44 & 22 & 1 & 15 & - & - & - & 55 \\
\hline $\begin{array}{l}\text { Konglo- } \\
\text { merat }\end{array}$ & & 23 & 42 & 7 & 3 & 18 & 3 & 2 & 2 & 82 \\
\hline
\end{tabular}

\subsection{Spaltenfüllungen}

Abgesehen von den beiden Basaltgängen kommt eine mit Calzit gefüllte $1 \mathrm{~m}$ breite Spalte am S-Hang des Yiron-Plateaus vor (Abb. 2). Nach einer Th/U-Datierung von Herrn HENNIG ist diese Füllung älter als $350 \mathrm{Ka}$. Vulkanische Komponenten wurden darin nicht gefunden. Die Füllung könnte damit älter sein als der Deckbasalt.

Am N-Hang der Baram-Platte sind nächst dem Talgrund nebeneinander zwei bis $3 \mathrm{~m}$ breite Spalten in Verlängerung des obengenannten Ganges und mit gleichem NNW-Streichen aufgeschlossen. Sie sind infolge Nachbruch von oben mit Basaltbrocken und einigen bearbeiteten Flintstückchen gefüllt (Abb. 2). Damit ist deren Altersstellung als postbasaltisch erwiesen. Außerdem zeigt sich damit an, daß die Basaltdecke ursprünglich wesentlich weiter verbreitet war.

\section{Morphogenese}

Die Morphogenese des Gebietes wird durch die ursprüngliche Entwässerung zum Mittelmeer (NIR 1970), den Einbruch des Jordan-Grabens sowie die tektonischen Bewegungen im Bereich der Mulde von 
Yiron bedingt. Überdies kommen vielleicht noch pleistzäne Klimaschwankungen hinzu.

Die Basalt-Decke taucht im Muldenbereich bereits etwas zum Jordan-Graben hin ab. Sicher aber ist, daß östlich Alma bereits ein Lavastrom in dieser Richtung geflossen ist. Auch der spärliche Kleinkies auf dem Plateau von Yiron deutet auf eine fluviatile Verlagerung von $\mathrm{W}$ nach $\mathrm{E}$.

Deutlich zeichnet sich die weitere Einschneidung des Dishon und Avod durch die Anlage eines Hochtals ab. Östlich von Yiron liegt eine Sohle in $630 \mathrm{~m} \mathrm{NN}$ mit generellem Absinken nach E. Seine Randzone ist wegen des allmählichen Anstieges nicht scharf begrenzbar (Abb. 1).

Lediglich südlich vom Plateau von Baram wurde in gleicher Höhe ein Flint-Schotter mit einzelnen kleinen Basalt-Geröllen gefunden. Hier könnte es sich um eine der Quellen handeln, aus der das Flintmaterial für die jüngeren Artefakte stammt. Die wenigen Basalt-Gerölle am S-Rand des Baram-Plateaus (Abb. 3, Profil 7) weisen ebenso wie die spärlichen Pyroxene (Abb. 4) darauf hin, daß auf der Höhe von Baram-Yiron die einstige W-Grenze der Basalt-Decken gelegen hat.

Die weitere Entwicklung des Dishon- (und Avod)Tales ist durch kerbenartige Eintiefung bis auf rd. 500 $\mathrm{m} N \mathrm{NN}$ in W gekennzeichnet. Sicherlich zeigt sich auch darin in erster Linie der Einfluß des JordanGrabens an.

Entlang dieses Talbereiches sind einige TerrassenReste vorhanden, so eine Konglomerat-Bank nördlich Alma in $500 \mathrm{~m}$ NN und nächst dem Talgrund ein Schotter mit zahlreichen Basalt-Geröllen. Auf der Gegenseite des Talgrundes liegt eine Mure, die ebenfalls Basaltbrocken führt. Wie Tab. 2 zeigt, fällt auch hier der Pyroxenanteil mit maximal 18\% recht spärlich aus.

\section{Die paläolithischen Funde} (von A. RONEN)

Die Lage der Paläolith-Funde ist in Abb. 6 dargestellt.

\subsection{Pre-basalt Artifacts}

Flint artifacts which predate the basalt flow were first discovered in February 1980, in a thick bed of red clay and gravel underlying the basalt on the eastern edge of the Yiron plateau (RONEN et al. 1980). The lithics, certainly of human origin, included cores, flakes and a few tools: a scraper, a bifacial point (?) and several retouched/utilised pieces. The small assemblage reflects a low degree of standadisation, as already noticed (RONEN et al. 1980). In April 1981 several test pits were dug with a back-hoe, for stratigraphical studies, at the eastern edge of the Yiron basalt. The pits yielded additional artifacts from depths of $1.8-2.5 \mathrm{~m}$ below the basalt base, which we present here:

- A thin flake in mint condition, with unprepared butt (Fig. 7: 1).

- A thick flake, slightly weathered, with "utilisation" retouch. The butt was broken off, possibly by deliberate direct retouch (Fig. 7: 2).

- A thick flake in mint condition, with dihedral butt and alternative retouch. The distal part is broken (Fig. 7: 3).

- A thin flake in mint condition, with facted butt and "utilisation" retouch (Fig. 7: 4).

The pre-basalt industry in the Upper Galilee is just beginning to show up. More data has to be gathered before we can even argue on the presence/absence of handaxes her. But ample evidence is already at hand for the presence of humans in the Upper Galilee prior to the Brunhes-Matuyama boundary.

\subsection{Post-basalt Industries}

Some 15 Acheulian open air sites/concentrations are known in the Upper Galilee. All but three are located on top, and near the margins, of basalt flows (the plateaux of Baram, Yiron and Dalton, and the Avot zone). Three concentrations are slightly off the basalt margin, perhaps due to the weathering away of the basalt (Fig. 6, the map). The artifacts are made of flint, in spite of the local abundance of basalt and limestone. Among thousands of artifacts examined, only two basalt handaxes were noticed, both in a weathered condition (RONEN et al. 1974: Fig. 6).

The artifacts are spread on top and across the entire thickness of the brown soil which covers the basalt. Test pits revealed that soil thickness reached $2 \mathrm{~m}$, and no stratigraphical differences could be distinguished in the industry. Hence, it may be concluded that the post-basalt industries are largely re-deposited.

All the concentrations except one include handaxes and belong without doubt to the Acheulian complex. The handaxes are composed mainly of Amygdaloids and rounded forms (Ovaloid and Discoidal). Cleavers are very few (RONEN et al. 1974). The flake industry is non-Levallois $(1 \%-3.7 \%)$, unfaceted $(14 \%-18.5 \%)$ and practically devoid of blades (0-4.3\%). The dominant tool types are knives, both backed and natural, denticulates, grattoirs and borers, including a small variety of borers (OHEL 1986: 144). 


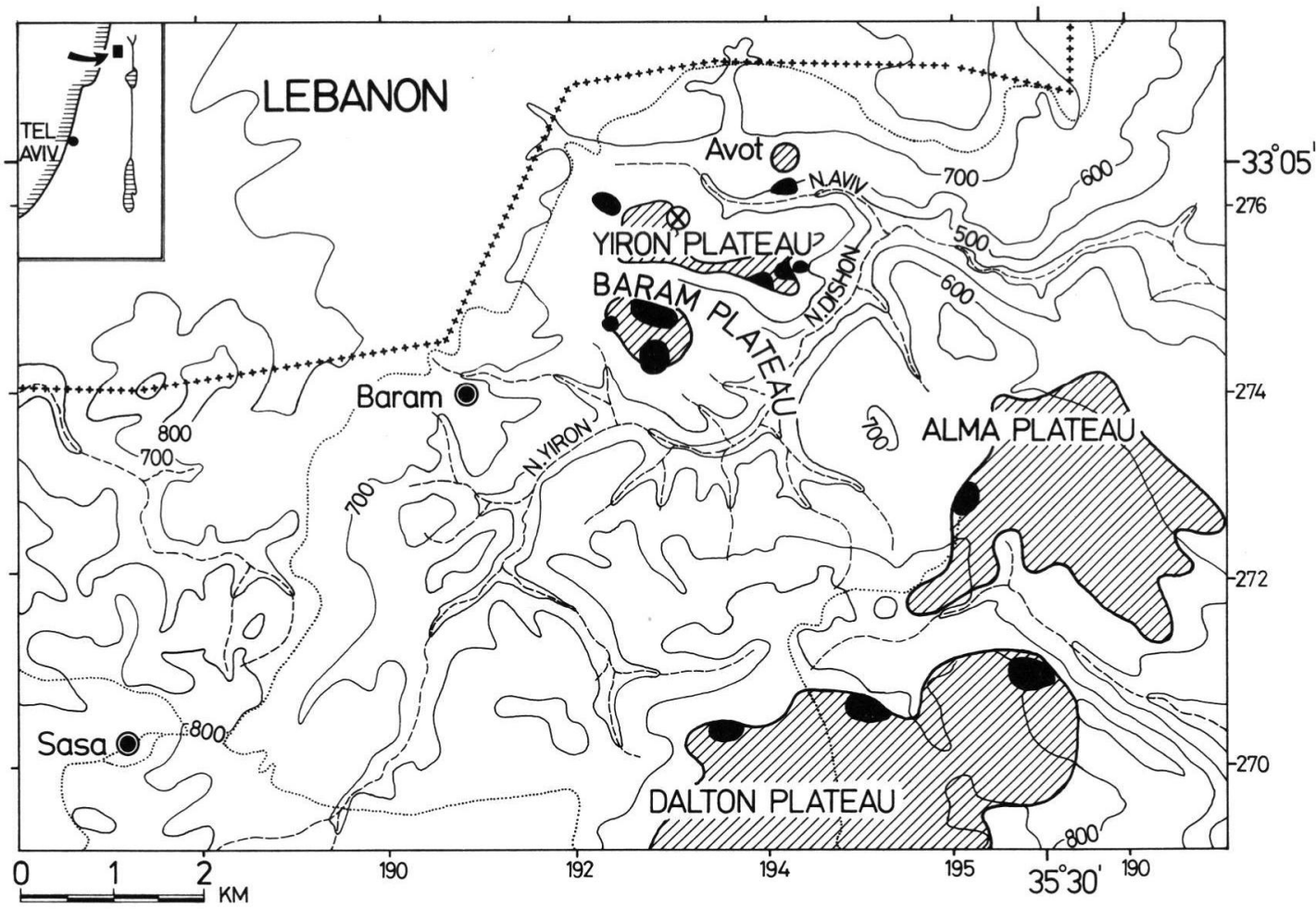

EZA BASALT COVER

POSTBASALTIC INDUSTRY

$\otimes$ PREBASALTIC INDUSTRY

Abb. 6: Lage der größeren Artefakt-Konzentrationen in Ober-Galiläa (nach RONEN et al., 1974 und OHEL, 1986). Fig. 6: Location of Major Lithic Concentrations in the Upper Galilee (after RONEN et al., 1974 and OHEL, 1986).

This Upper Galilee Acheulian differs from the other Acheulian occurrences known at present on the coastal plain (RONEN et al. 1974), on the Golan (GOREN 1979) or in the caves of Tabun and UmmQatafa. In our opinion, this originality may reflect a specific time bracket rather than a regional facies (the environmental conditions on the Golan are quite similar to those of Upper Galilee).

The single site/concentration which does not contain handaxes or other bifacial tools is the one at Avot (OHEL 1986; Site A 63). It is one of the assemblages which are found on limestone, not far from the edge of the basalt flow. The Avot assemblage is more "primitive" than the others, and like them, of a nonLevallois technique. Hence, it can not be considered of Middle Palaeolithic age. It may have been a workshop, contemporary with the Acheulian occurences; but the absence even of unfinished bifaces is hard to explain. The hypotheses of „winter dwelling” (OHEL 1986) similarly does not explain the complete disappearance of bifaces. The Avot site may, in out opinion, be older than the other concentrations, perhaps pre-dating the basalt flow.

\section{Stratigraphische Situation}

Der Versuch mit von K/Ar-Daten den Deckbasalt zu datieren, schlug wegen dessen Anwitterung fehl. Mit Hilfe der Paläomagnetik konnte jedoch gezeigt werden, daß dieses Material revers magnetisiert ist, $d$. h. es liegt zeitlich vor der Brunhes/Matuyama-Grenze.

Die Bodensedimente von Yiron können damit ebenfalls in das frühe Pleistozän eingestuft werden und möglicherweise als Folge einer kaltzeitlichen Rhexistasie im ältesten Pleistozän interpretiert werden.

Einen gewissen Datierungsansatz liefert ferner die am Grabenrand gelegene, $\mathrm{rd}$. $40 \mathrm{~km}$ entfernte Station von Ubaidiya. Basaltschutt und die stabile Pyroxenführung lassen sich vom benachbarten Yarmouk-Deckbasalt ableiten (Abb. 4). Dieser Basalt wird um 600 bis $650 \mathrm{Ka}$ eingestuft. Folglich sollte die Station Ubaidiya etwas jünger eingeordnet werden können als die in Yiron.

Aufgrund dieser Überlegung dürfte das präbasaltische Paläolithikum von Yiron neben Evron und Ubaidiya zu den ältesten Spuren des Menschen im Vorderen 

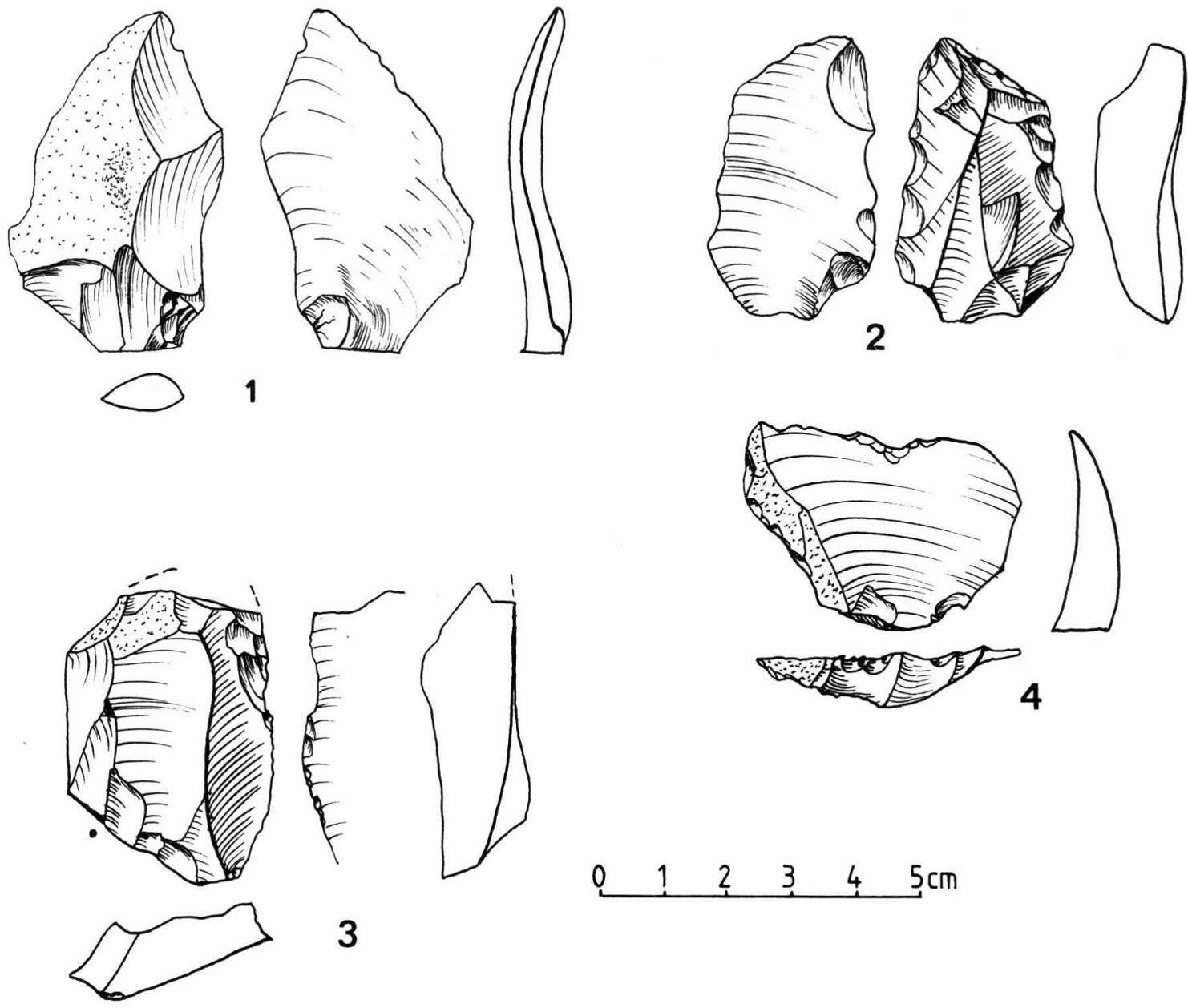

3

Abb. 7: Präbasaltische Flint-Artefakte bei Yiron.

Fig. 7: Prebasaltic flint artifacts beneath the Yiron basalt.

Orient gehören. Aber auch die postbasaltische Faustkeilkultur bei Yiron und Baram muß dann als relativ alt eingestuft werden, wenn die Beziehung zur Hochtal-Zone berechtigt ist (Abb. 6); denn erst danach ändert sich der Typ der Talbildung, der vom JordanGraben gesteuert werden konnte.

\section{Schriftenverzeichnis}

Boenigk, W., BrunNaCKeR, K., Tillmanns, W. \& RoNEN, A. (1985): Die Äolianite in der nördlichen Küstenzone von Israel - Genese, Stratigraphie und Klimageschichte. - Quartär, 35/36: 113-140, 13 Abb., 3 Tab.; Erlangen.
FLEXNER, A. (1964): Late Cretaceous palaeogeography of northern Israel and its significance for the Levant geology. - Palaeogeogr., Palaeoclimatol., Palaeooecol., 10 (4): 293-316.

GoreN, N. (1979): An Upper Acheulian industry from the Golan Heights. Quartär, 29/30: 105-121; Erlangen.

Greenberg, M. (1960): Mineralogy and Petrography of the Lower Cretaceous of Makhtesh Hathira. - Geol. Dept. Hebrew Univ., Jerusalem (Unpublished M. Sc. theses. In Hebrew).

HoRowiTz, A. (1979): The Quaternary of Israel. - New York (Academic Press). 
ISKANDAR, E. (1985): Sedimentpetrographische Untersuchungen an Rotlehmen aus Obergaliläa und an Sedimenten der Unteren Serie der „Ubaidiya-Formation, Israel. - 66 S.; Geol. Inst. der Univ. zu Köln (unveröff. Diplomarbeit).

NACHMIAS, J. (1969): Source rocks of the Saqiye Group sediments in the coastal plain of Israel - a heavy mineral study. - Israel J. Earth Sci., 18: 1-16; Jerusalem.

NEEV, D. (1960): A pre-Neogene erosion channel in the Southern Coastal Plain of Israel. - Bull. geol. Sur., 25: 1-21; Jerusalem.

NIR, A. (1970): Geomorphology of Israel. - Jerusalem (Academon).

OHel, M. Y. (1986): The Acheulean of the Yiron Plateau, Israel. - BAR Intern., Series 307; Oxford.

OPPENHEIM, M. J. (1962): The geology of the southeastern Galilee lava fields. - Bull. Res. Counc. Israel, 11G: 58-84; Jerusalem.
RONEN, A., GILEAD, D., BRUdeR, G. \& MELleR, P. (1974): Notes on the Pleistocene Geology and Prehistory of the Central Dishon Valley, Upper Galilee, Israel. - Quartär, 25: 13-23; Erlangen.

Ronen, A., Inbar, M., Klein, M. \& BRUNNACKer, K. (1980): Artifact-bearing gravels beneath the Yiron basalt. - Israel J. Earth Sciences, 29: 221-226, 6 Abb.; Jerusalem.

VROMAN, A. (1944): The petrology of sandy sediments of Palestine. - Bull. Geol. Dept., Heb. Univ. 5; Jerusalem.

ZOHARY, A. (1959): In HorowTTZ, A. (1979): The Quaternary of Israel. - New York (Academic Press).

Manuskript eingegangen am 5. 7. 1988. 